MADPH-00-1152

JANUARY, 2000

\title{
CP VIOLATING PHASES AND THE DARK MATTER PROBLEM*
}

\author{
TOBY FALK \\ Department of Physics, University of Wisconsin, Madison, WI 53706, USA
}

\begin{abstract}
New CP violating phases in the MSSM can affect both the abundance and detection of neutralino dark matter. We discuss the effect of including cosmological constraints in the limits on new sources of CP violation in the MSSM and the effects of new $\mathrm{CP}$ violating parameters on dark matter densities and detection.
\end{abstract}

\section{CP Violation in Supersymmetry}

This last year has seen a lot of work on CP violating phases in supersymmetryhow to constrain the phases, how to measure them, how to avoid the same constraints, and the extent to which $\mathrm{CP}$ violation can spoil predictions appropriate in the absence of CP violation in the SUSY parameters. Today I will discuss some of the cosmological consequences of $\mathrm{CP}$ violating phases in the MSSM, and in particular, their effect on the abundance and detection of SUSY dark matter.

The Supersymmetric Standard Model contains many new potential sources of $\mathrm{CP}$ violation beyond that of the standard model. In particular, the supersymmetric Higgs mixing mass $\mu$, the gaugino masses $M_{i}$, the scalar trilinear couplings $A_{i}$ and the SUSY breaking scalar Higgs mixing parameter $B \mu$ can all in principle be complex. However, not all the phases are physical, and depending on the model, some or most can be removed by field redefinitions. The remaining sources for $\mathrm{CP}$ violation are experimentally constrained, primarily due to their contributions to the Electric Dipole Moments (EDMs) of the electron and neutron, and in particular, the EDM of the mercury atom ${ }^{199} \mathrm{Hg}$.

\section{2 mSUGRA Constraints}

In minimal Supergravity (mSUGRA), the large number of relations between the SUSY parameters reduces the set of $\mathrm{CP}$ violating phases to just two:

*PRESENTED AT COSMO99: 3RD INTERNATIONAL CONFERENCE ON PARTICLE PHYSICS AND THE EARLY UNIVERSE, TRIESTE, ITALY

cosmo99proc: submitted to World Scientific on November 29, 2018 
$\theta_{\mu}$, associated with the Higgs mixing mass $\mu$, and $\theta_{A}$, a common trilinear parameter phase. These phases then appear in the low energy Lagrangian in the neutralino and chargino mass matrices (in the case of $\theta_{\mu}$ ) and in the left-right sfermion mixing terms (both $\theta_{\mu}$ and $\theta_{A}$ ). The new sources for $\mathrm{CP}$ violation then contribute to the EDMs of standard model fermions, and the tight experimental constraints on the EDMs of the electron, neutron and mercury atom place severe limits on the sizes of $\theta_{\mu}$ and $\theta_{A}$ Q .

The EDMs generated by $\theta_{\mu}$ and $\theta_{A}$ are sufficiently small if either 1 ) the phases are very small $\left(\lesssim 10^{-2}\right)$, or 2$)$ the SUSY masses are very large $(\mathcal{O}$ (a few $\mathrm{TeV}))$, or 3$)$ There are large cancellations between different contributions to the EDMs. In mSUGRA, option 2) is forbidden by the relic density constraints, as we'll show next. Condition 3), large cancellations, does naturally occur in mSUGRA models over significant regions of parameter space, including in the body of the cosmologically allowed region with $m_{1 / 2}=\mathcal{O}(100-400 \mathrm{GeV})$. These cancellations relax the constraints on the phases, but the limit on $\theta_{\mu}$ remains small, $\theta_{\mu} \lesssim \pi / 10$.

To see why option 2) is cosmologically forbidden, recall that the SUSY phases contribute to the electron EDM, for example, via processes of the following type:

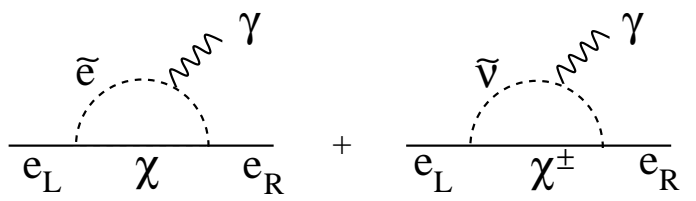

where selectrons and sneutrinos appear in the loop. These contributions diminish as the sfermion masses are increased, but this also shuts off neutralino annihilation in the early universe, which is dominated by sfermion exchange as in Fig. 1, and hence increases the neutralino relic abundance. In Fig. 2a we denote 3 by light shading the region of the $m_{1 / 2}-m_{0}$ parameter space with a relic neutralino abundance in the preferred range $0.1 \leq \Omega_{\tilde{\chi}} h^{2} \leq 0.3$. The upper bound on $\Omega_{\tilde{\chi}} h^{2}$, coming from a lower limit of $12 \mathrm{Gyr}$ on the age of the universe, then limits the extent to which one can turn off the electron EDMs by raising the sfermion masses. The combination of cosmological with EDM

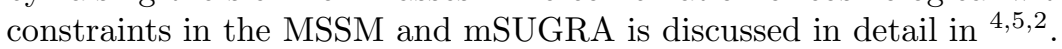

To demonstrate the combined limits on $\theta_{\mu}$ and $\theta_{A}$ in mSUGRA, we plot in the $\left\{\theta_{\mu}, \theta_{A}\right\}$ plane the minimum value of $m_{1 / 2}$ required to bring the EDMs of both the electron and the mercury atom ${ }^{199} \mathrm{Hg}$ below their respective experimental constraints (Fig. 2 $\mathrm{b}$ ). These experiments currently provide the 


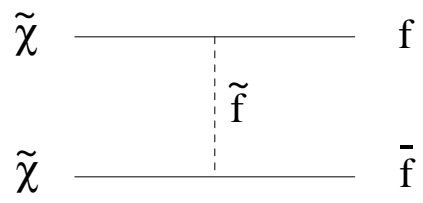

Figure 1. Sfermion exchange dominates $\chi$ 's annihilation.

tightest bounds on the SUSY phases ${ }^{a}$. Here we've fixed $\tan \beta=2, A_{0}=300$ $\mathrm{GeV}$ and $m_{0}=100$ and scanned upwards in $m_{1 / 2}$ until the experimental constraints are satisfied. Due to cancellations, the EDMs are not monotonic in $m_{1 / 2}$; however, there is still a minimum value of $m_{1 / 2}$ which is allowed. In the absence of coannihilations, there is an upper bound on $m_{1 / 2}$ of about 450 $\mathrm{GeV}$ (though slightly smaller for this $m_{0}$ ); an analogous figure to Fig. 2a for $\tan \beta=2$ shows that coannihilations increase the bound to about $600 \mathrm{GeV}$. Comparing with Fig. 2a, we see that zone V is cosmologically forbidden, and that the effect of including coannihilations is to allow zone IV, which was formerly excluded.

The bowing to the right of the contours in Fig. 2 is a result of cancellations between different contributions to the EDMs 6 , and we can see that the effect is to relax the upper bound on $\theta_{\mu}$ by a factor of a few. As we increase $A_{0}$, the extent of the bowing increases, and larger values of $\theta_{\mu}$ can be accessed. This loophole to larger $\theta_{\mu}$ is limited by the diminishing size of the regions in which there are sufficient cancellations to satisfy the EDM constraints. In general, the regions of cancellation for the electron EDM are different than those for the ${ }^{199} \mathrm{Hg} \mathrm{EDM}$, and the two regions do not always overlap. As $\theta_{\mu}$ is increased, the sizes of the regions of sufficient cancellations decrease; in Fig. 2 , the width in $m_{1 / 2}$ of the combined allowed region near the $\theta_{\mu}$ upper bound is $40-80 \mathrm{GeV}$, which on a scale of $200-300 \mathrm{GeV}$ is reasonably broad. Larger $A_{0}$ permits larger $\theta_{\mu}$, but the region of cancellations shrinks so that a careful adjustment of $m_{1 / 2}$ becomes required to access the largest $\theta_{\mu}$. At the end of the day, values of $\theta_{\mu}$ much greater than about $\pi / 10$ cannot satisfy the EDM constraints without significant fine-tuning of the mass parameters. At larger values of $\tan \beta$, the upper bound decreases roughly as $1 / \tan \beta$. See 3 for more details on the status of EDM and cosmological constraints on $\mathrm{CP}$ violating

${ }^{a}$ The extraction of the neutrep EDM from the SUSY parameter space is plagued by significant hadronic uncertainties 2, so that the inclusion of the neutron EDM constraint does not improve the limits when the uncertainties in the calculated neutron EDM are taken into account

cosmo99proc: submitted to World Scientific on November 29, 2018 

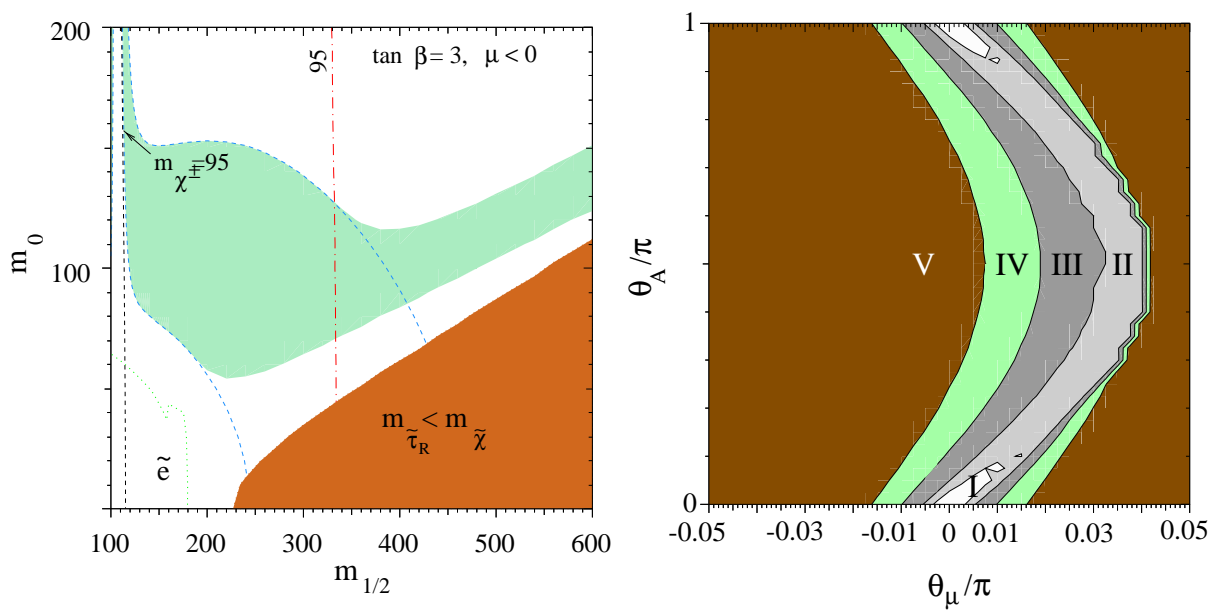

Figure 2. a) The light-shaded area is the cosmologically preferred region with $0.1 \leq \Omega \sim{ }_{\chi} h^{2} \leq 0.3$. In the dark shaded regions in the bottom right of each panel, the LSP is the $\tilde{\tau}_{R}^{\chi}$, leading to an unacceptable abundance of charged dark matter. Also shown are chargino and Higgs isomass contours and an indication of the slepton bound from LEP. b) Contours of $m_{1 / 2}^{\min }$, the minimum $m_{1 / 2}$ required to bring both the electron and Hg EDMs below their respective experimental bounds, for $\tan \beta=2, m_{0}=130 \mathrm{GeV}$, and $A_{0}=300 \mathrm{GeV}$. The central light zone labeled "I" has $m_{1 / 2}^{\min }<200 \mathrm{GeV}$, while the zones labeled "II", "III", and "IV" correspond to $200 \mathrm{GeV}<m_{1 / 2}^{\min }<300 \mathrm{GeV}, 300 \mathrm{GeV}<m_{1 / 2}^{\min }<450 \mathrm{GeV}$, $450 \mathrm{GeV}<m_{1 / 2}^{\min }<600 \mathrm{GeV}$ and $m_{1 / 2}^{\min }>600 \mathrm{GeV}$, respectively. Zone $\mathrm{V}$ is therefore cosmologically excluded.

phases in mSUGRA.

\section{Large Phases}

Much of the work on SUSY CP violation in the last year has been inspired by the hope of having large $(\mathcal{O}(1))$ phases, and there have been several suggestions as to how this might be achieved while satisfying the stringent constraints from the EDMs. First, the presence of cancellations between different contributions to the fermion EDMs (which recently has dubbed the "cancellation mechanism" 6 ) has been used to motivate interest in large phases, although as we have seen in the last section, in mSUGRA, cosmological considerations limit the extent to which cancellations can free the phases. If gaugino mass unification is broken, then there are two additional phases in the model, namely the relative phases between $M_{1} M_{2}$ and $M_{3}$. More phases then lead to more opportunities for cancellations 6 . There may be hints that

cosmo99proc: submitted to World Scientific on November 29, 2018 
string theory can provide (small) regions with large phases in models without gaugino mass unification 10 .

Alternatively, note that the one-loop diagrams contributing to the fermion EDMs only contain first generation sfermions. Hence in models in which the first (or first two) generation sfermions are extremely heavy 13 , the EDMs are suppressed even with $\mathcal{O}(1)$ phases. Of course if the LSP neutralino is gauginolike, the third generation sfermions must be quite light in order to satisfy the relic density constraints. The phases in these models are still not completely unconstrained howeyer. The third generation sfermions can contribute to the EDMs at two loops 8 , and further, phases in the stop sector (i.e. $\theta_{\mu}, \theta_{A_{t}}$ ) enter radiatively into the Higgs potential $\mathbf{l}$ and induce a phase misalignment between the Higgs vevs, and this can potentially introduce visible effects. Both the latter effects are particularly important at large $\tan \beta$.

\section{Neutralino Relic Density}

As originally shown in $\mathrm{A}, \mathrm{CP}$ violating phases can have a large effect on the relic density of the Lightest Supersymmetric Particle (LSP) in SUSY models. This occurs because the dominant annihilation channel for a gaugino-like neutralino (Fig. 1) exhibits "p-wave suppression". That is, if one expands the thermally averaged annihilation cross-section at freeze-out in powers of $\left(T / m_{\tilde{\chi}}\right)$,

$$
\left\langle\sigma_{\tilde{\chi} \tilde{\chi}} v\right\rangle=a+b\left(T / m_{\tilde{\chi}}\right)+\mathcal{O}\left(T / m_{\tilde{\chi}}\right)^{2}
$$

the zeroth order term $a$ is suppressed 11 by $m_{f}^{2}$. This suppresses the annihilation rate in the early universe, and enhances the $\chi$ relic abundance, by more than an order of magnitude. However, in the presence of left-right sfermion mixing and $\mathrm{CP}$ violating phases, the zeroth order term has a piece

$$
a \approx \frac{g_{1}^{4}}{32 \pi} \frac{m_{\tilde{\chi}}^{2}}{\left(m_{\tilde{f}}^{2}+m_{\tilde{\chi}}^{2}-m_{f}^{2}\right)^{2}} Y_{L}^{2} Y_{R}^{2} \sin ^{2} 2 \theta_{f} \sin ^{2} \gamma_{f}+\mathcal{O}\left(m_{f} m_{\tilde{\chi}}\right)
$$

where $\theta_{f}$ is the sfermion mixing angle and $\gamma_{f}=\operatorname{Arg}\left(A_{f}^{*}+\mu \tan \beta\right)$. For significant $\theta_{f}$ and $\gamma_{f}$, this results in a dramatic increase in the annihilation rate and decrease in the $\chi$ relic density, and it weakens the cosmological upper bound on $m_{\tilde{\chi}}$ (in the absence of coannihilations) from $\sim 250 \mathrm{GeV}$ to $\sim 650 \mathrm{GeV}$. In mSUGRA, however, neutralino annihilation is primarily through sleptons into lepton pairs, and the mixing angles $2 \theta_{f}$ are typically very small. The effect of $\mathrm{CP}$ phases on the $\chi$ annihilation in mSUGRA is therefore negligiblel. This is also generally true in models where the sfermion mixing angles are small; e.g. for a string inspired model, see 12.

cosmo99proc: submitted to World Scientific on November 29, 2018 
Phases also potentially affect masses and couplings of the neutralino and charginos, stop and Higgs particles, as well as providing mixing between the scalar and pseudoscalar Higgs 14 , and these can effect the neutralino relic density, particularly for a Higgsino-type neutralino.

\section{Neutralino Direct Detection}

Phases can also affect the direct detection of relic neutralinos. In direct detection schemes, relic neutralinos elastic scatter off of nuclei in a target material, depositing a detectable amount of energy. The low-energy effective four-Fermi Lagrangian for neutralino-quark interactions takes the form

$$
\begin{aligned}
\mathcal{L}= & \bar{\chi} \gamma^{\mu} \gamma^{5} \chi \bar{q}_{i} \gamma_{\mu}\left(\alpha_{1 i}+\alpha_{2 i} \gamma^{5}\right) q_{i}+\alpha_{3 i} \bar{\chi} \chi \bar{q}_{i} q_{i}+\alpha_{4 i} \bar{\chi} \gamma^{5} \chi \bar{q}_{i} \gamma^{5} q_{i}+ \\
& \alpha_{5 i} \bar{\chi} \chi \overline{q_{i}} \gamma^{5} q_{i}+\alpha_{6 i} \bar{\chi} \gamma^{5} \chi \bar{q}_{i} q_{i},
\end{aligned}
$$

The coefficients $\alpha_{2}$ and $\alpha_{3}$ contribute to spin-dependent and spin-independent neutralino-nщcleon scattering, respectively. The phases enter into the coefficients $\alpha_{i}$ 15. 16 .
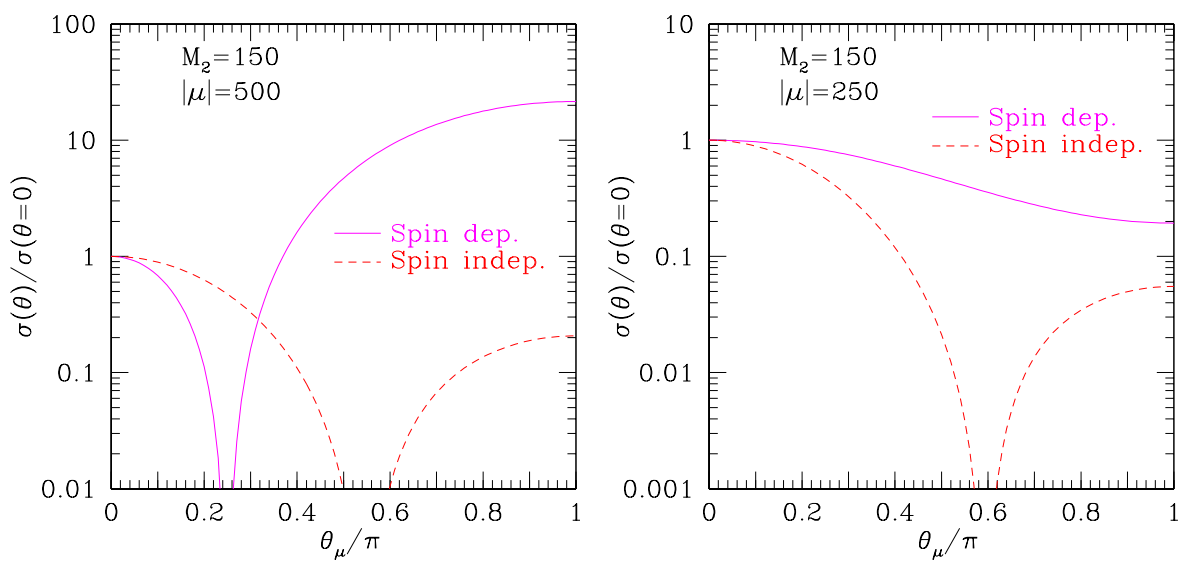

Figure 3. Direct detection rates as a function of $\theta_{\mu}$ for scattering off of ${ }^{19} \mathrm{~F}$ and $\left.\mathrm{b}\right){ }^{73} \mathrm{Ge}$. Here $\tan \beta=3$ and $m_{0}=100 \mathrm{GeV}$.

Due to cancellations in the scattering rates, phases can produce a significant effect on the detection rate. In Fig. 3, we show the neutralino-nucleus elastic scattering cross-section as a function of $\theta_{\mu}$, displaying separately the spin-dependent and spin-independent contributions, for two target nuclei, ${ }^{19} \mathrm{~F}$ and ${ }^{73} \mathrm{Ge}$. Dramatic reductions in the spin-independent cross-section occur near $\theta_{\mu}=0.6 \pi$ in both cases, and near $\theta_{\mu}=0.25 \pi$ for the spin-dependent rate 
for ${ }^{19} \mathrm{~F}$. Of course these large values of $\theta_{\mu}$ are excluded in mSUGRA, as we have seen above, although in more general models one can tune the other parameters (e.g. the trilinear parameters $A_{i}$ ) in order produce the cancellations necessary to satisfy the EDM constraints, as we have done in Fig. 3 .
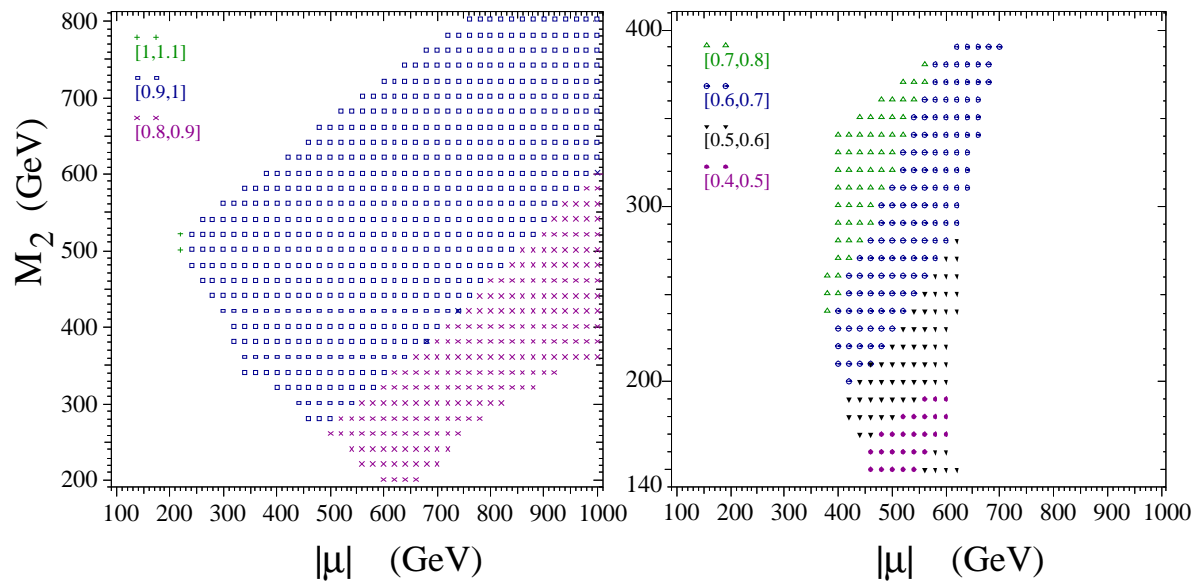

Figure 4. Ratios of direct detection rates for scattering off of ${ }^{19} \mathrm{~F}$ with and without $\mathrm{CP}$ violation, for a) $\theta_{\mu}=\pi / 8, \theta_{A}=3 \pi / 8$, b) $\theta_{\mu}=\pi / 4, \theta_{A}=\pi / 2$

In Fig. 14 we perform a scan over MSSM parameters $M_{2}, \mu, A$ and $m_{0}$ for fixed phases $\theta_{\mu}$ and $\theta_{A}$ and $\tan \beta=3$ and compute the ratio of total scattering cross-sections with and without phases, for scattering off of ${ }^{19} \mathrm{~F}$. We haven't chosen the phases to lie in the dips of Fig. 3, so Fig. 4 isn't intended to indicate the maximum possible effect of the phases. Rather, we hope display a more typical result and to demonstrate the variation of the effect of the phases as a function of the parameters $\mu$ and $M_{2}$. We see that reductions in the rate up to $\% 50$ and enhancements by up to \%10 occur over much of the $M_{2}-\mu$ parameter space. The plotted points all satisfy the EDM constraints. There is one caveat to bear in mind: because we have done a scan over parameters, we have found the (small) regions of parameter space satisfying the EDM bounds for these large values of the phases. These regions are not generic, and in fact are uncomfortably tuned. Thus we simply take these plots as an existence proof that CP-violating phases can have a significant effect on the direct detection of neutralino dark matter. 


\section{Summary}

New sources of CP violation are present in the MSSM which are not present in the standard model. In mSUGRA, cosmological bounds on $m_{1 / 2}, m_{0}$ and $m_{\tilde{\chi}}$ combine with limits on the Electric Dipole Moments of the electron and ${ }^{199} \mathrm{Hg}$ to constrain $\theta_{\mu} \lesssim \pi / 10$, while $\theta_{A}$ remains essentially unconstrained. In general models, phases can affect neutralino annihilation, so that the cosmological upper bound on $m_{\tilde{\chi}}$ increases from 250 to $650 \mathrm{GeV}$. Phases can also affect neutralino direct detection rates, typically reducing them by a factor $\sim 2$, but orders of magnitude in parts of the parameter space.

\section{Acknowledgments}

The work of T.F. was supported in part by DOE grant DE-FG02-95ER40896, and in part by the University of Wisconsin Research Committee with funds granted by the Wisconsin Alumni Research Foundation.

\section{References}

1. M. Dugan, B. Grinstein and L. Hall, Nucl. Phys. B255, 413 (1985); Y. Kizukuri \& N. Oshimo, Phys. Rev. D45 (1992) 1806; D46(1992) 3025.

2. T. Falk, K.A. Olive, M. Pospelov and R. Roiban, hep-ph/9904393.

3. J. Ellis, T. Falk, and K.A. Olive, Phys. Lett. B444 (1998) 367.

4. T. Falk, K.A. Olive and M. Srednicki, Phys.Lett. B354 (1995) 99; T. Falk and K.A. Olive, Phys. Lett. B439 (1998) 71.

5. T. Falk and K.A. Olive, Phys. Lett. 375 (1996) 196.

6. T. Ibrahim, P. Nath, Phys. Rev.D58 (1998) 111301; Erratum-ibid.D60 (1999) 099902.

7. M. Brhlik, G. J. Good and G.L. Kane, Phys. Rev. D59 (1999) 115004.

8. D. Chang, W. Keung, A. Pilaftsis, Phys.Rev.Lett. 82 (1999) 900.; Erratum-ibid.83 (1999) 3972; D. Chang, W. Chang and W. Keung, hepph/9910465.

9. A. Pilaftsis, Phys. Rev. D 58 (1998) 096010; A. Pilaftsis, Phys. Lett. 435B (1998) 88; D. A. Demir, hep-ph/9901389; D. A. Demir, hep$\mathrm{ph} / 9905571$.

10. M. Brhlik, L. Everett, G.L. Kane and J. Lykken, Phys.Rev.Lett. 83 (1999) 2124; hep-ph/9908326.

11. H. Goldberg, Phys. Rev. Lett. 50 (1983) 1419.

12. S. Khalil and Q. Shafi, hep-ph/9904448.

cosmo99proc: submitted to World Scientific on November 29, 2018 
13. S. Dimopoulos and G.F. Giudice, Phys. Lett. B357 (1995) 573 ; A. Cohen, D.B. Kaplan and A.E. Nelson, Phys. Lett. B388 (1996) 599 ; A. Pomarol and D. Tommasini, Nucl. Phys. B488 (1996) 3.

14. A. Pilaftsis and C.E.M. Wagner, Nucl.Phys. B553 (1999) 3.

15. T. Falk, A. Ferstl, and K.A. Olive, Phys. Rev. D59 (1999) 055009; T. Falk, A. Ferstl, and K.A. Olive, hep-ph/9908311.

16. U. Chattopadhyay, T. Ibrahim, and P. Nath, Phys. Rev. D60 (1999) 063505; P. Gondolo and K. Freese, hep-ph/990839d; S.Y. Choi, hep$\mathrm{ph} / 9908397$.

cosmo99proc: submitted to World Scientific on November 29, 2018 